\title{
27. COMMISSION DES ÉTOILES VARIABLES
}

Président: M. R. S. Dugan, Princeton University Observatory, Princeton, N.J., U.S.A.

Vice-Président: M. Grouilier.

Membres: MM. Banachiewicz, L. Campbell, Cox, Danjon, de Roy, Einbu, Gadomski, Gaposchkin, Gerasimovich, Graff, Guthnick, Mlle Harwood, MM. Hertzsprung, Hoffmeister, Mme H. B. Hogg, MM. Jacchia, Jordan, Joy, Kopal, Kordylewski, Kukarkin, Lacchini, Lindley, Ludendorff, McLaughlin, Merrill, Mitchell, A. V. Nielsen, Pearce, D. B. Pickering, Prager, Ryves, Shapley, Silva, Steavenson, Stebbins, Stein, E. Strömgren, ten Bruggencate, Tiercy, Tsesevich, Voûte, Walter.

I. The Commission again subscribes to a number of the good resolutions it has made in the past, for example, to follow the almost universal practice of counting the observed times, either in decimals of a day or in hours and minutes, from Greenwich mean noon, even though one is convinced that the rest of the world should adopt U.T.; and to prepare a chart, identifying the variable and the comparison stars, to form a part of the discovery announcement of a variable which cannot be easily identified through a Durchmusterung number and which is bright enough to invite further observation.

2. In accordance with the decision of the Commission, the revision of Nijland's lists of stars for which co-operative observing is considered especially desirable was delegated to Leon Campbell. The list will be published as an appendix to this report and may be obtained in reprint form from the General Secretary.

The report concerning unpublished observations, which was undertaken by Grouiller, will be published soon in Acta Astronomica. Reprints may be secured from the General Secretary. Considering the difficulties encountered in making the list at all complete, Prof. Grouiller suggests consideration of the advisability of continuing this project and later publishing a supplementary list.

A prospect of serious duplication in the calculation of tables for eclipsing variables in Russell's method of solution has been averted by an agreement whereby W. Tsesevich takes the responsibility for new. "darkened" tables, and J. E. Merrill for "uniform" tables.

3. Campbell reports on the work of the variable star associations in part as follows: Between four and five hundred variable stars of long period have been continuously followed during the past twenty-five to thirty years. We now have data that are sufficiently reliable to study forms of light curves and changes in period (Sterne and Campbell, Tercentenary Papers, H.A., 105, 459), but more data are necessary to check up on these and to note additional peculiarities in curves. We have also been able to lay down a sort of classification of the various longperiod variables. For such stars as SS Cyg, Z Cam, RR Tau, and V Sge we cannot obtain too much data. More stars should be added to the observing lists-none taken off ! More can be added once additional sequences and charts are available. About fifty new sequences are now being prepared at Harvard. When these are completed the various associations will be asked whether they wish to add some of these variables to their observing lists. (From this letter as well as from several others it is apparent that these associations profit greatly by keeping in close touch with each other and maintaining a mutual understanding on their programmes.) 
4. The revision of sequences by Mitchell (Publ. Leander McCormick Obs., Vol. 6) and the preparation of new and improved charts have provided a homogeneous system for the observation of long-period variables. Together with those already determined for stars brighter than the $1^{3 t h}$ magnitude at minimum, there are now sequences on one system for some 450 long-period variables. A decided increase in the accuracy of the observations has already resulted.

5. Stein writes that Series IX of Hagen's Atlas Stellarum Variabilium will soon appear in a less expensive form than previous volumes. It will contain 4I charts with 44 variable stars. Photovisual magnitudes have been determined from the polar sequence with a thermoelectric photometer.

Attention is drawn by Nielsen to the working list of the Scandinavian Astronomical Society published in Nordisk Astronomisk Tidsskrift, I937, II2; and to the need for international co-operation in obtaining a continuous light curve of RY Tau.

6. The Central Bureau for Variable Stars in U.S.S.R. reports good progress on the Plan of Investigation of Unstudied Variable Stars. Of the 176 fields into which the sky is divided according to this plan, responsibility for 78 has been assumed by thirty astronomers. The number of visual observations (on I500 stars) already exceeds 25,000 , and more than I000 plates have been taken. Visual magnitudes have been determined for comparison stars of 120 variables, and photographic magnitudes for about the same number. All the observers taking part in the plan live in the northern hemisphere and the Bureau wishes attention drawn to the fact that the whole undertaking, as a statistical project, will suffer greatly if the stars south of declination $-30^{\circ}$ remain unobserved. Several members urge the importance of co-operation in this plan in other countries. The Engelhardt $\mathrm{Ob}-$ servatory has assembled a card catalogue of eclipsing variables (about $\mathbf{1}_{500}$ ) with full bibliographical data.

7. The second volume of the GuL (Cyg to Oph) was published in 1936 and the preparation of the third volume has been started by Prager.

Prager's second list (Erg. A.N. ro, Nr. I, I937) brings up to date the count of stars announced as variable but not yet "named" by the AG-commission. Like his former list (ibid. 9, Nr. 3, I934) and the still earlier one by Zinner (ibid. 8, Nr. I, I929), it omits the stars of small range listed by Miss Güssow and Guthnick in their Katalog lichtelektrisch gemessener Sterne (K.V.B.B. Nr. 8, I930); and in general the stars in globular clusters, the Magellanic Clouds, and extra-galactic nebulae. Students are now in a better position for statistical studies, and observers can make a more complete check on the newness of their discoveries before publication. Some may not have noticed that the Benennungsliste now give, in the notes, the Prager (P) numbers of those stars that are promoted from provisional to definitive designations. A second edition of the Tabellen zur Nomenklatur der veränderlichen Sterne (K.V.B.B. Nr. 2, 1927), so useful in cross references, for which there is considerable inquiry, still lies in the future.

The tracing of references in the literature will be materially facilitated by the plan of republishing in the Astronomische Nachrichten discovery lists from other publications, so that such stars may be included in the system of provisional designation (e.g. 470.I934 Aur). Authors will be able to assist in this praiseworthy plan by seeing to it that Prof. Kobold receives copies of such lists promptly. This, of course, need not interfere at all with national, institutional, or personal systems of designation.

8. Guthnick writes that the present practice of the AG-commission is to make the following minimum requirements for the "Benennung" of stars announced as 
variable: firstly, the data make the variation unquestionable and are sufficient for statistical purposes; secondly, the statements concerning position are sufficient for sure identification (in the case of non-catalogue stars, positions should be given to $I^{\prime}$ and $o^{\prime} \cdot I$ ). In the first group of conditions are included range in magnitude and type of variation. "Kurzperiodisch" is not regarded as sufficiently "eindeutig" for statistical purposes. "Irregular", on the other hand, is regarded as a sufficient characterization, but it is desirable, when possible, that it be stated whether it is a red irregular variable or one of some other kind.

9. The increasing participation of spectroscopists in the observation of variable stars is very encouraging. Dr Moore reports that there are about one hundred eclipsing variables on the radial velocity programmes of eight observatories with which his sub-committee is in communication. At Potsdam and Babelsberg it is planned to make "gleichzeitige lichtelektrische Beobachtungen der Helligkeit, Objektivprismenaufnahmen, die einen möglichst grossen Spektralbereich umfassen, und Serienaufnahmen des Spektrums mit Spaltspektrographen zur Bestimmung der Radialgeschwindigkeit, von einigen ausgewählten Sternen. Es soll damit die Frage parallel laufender Schwankungen der Helligkeit, Farbtemperatur, und der Radialgeschwindigkeit weiter geklärt werden."

Among the larger surveys is that of the radial velocities of I26 Cepheids just completed and published ( $A p . J .86,363$, I937) by Joy. This leaves only about 35 known Cepheids, mostly fainter than the 14th magnitude, north of declination $-40^{\circ}$ for which no radial velocity observations are now available. Merrill states that of the long-period variables about 285 , mostly brighter than magnitude Ir, have now been observed near maximum for radial velocity. A few score additional stars are probably within reach of the roo-inch telescope. Joy has scattered spectrographic observations of 70 stars of the RV Tauri and intermediate types with periods between 50 and I50 days, and of 70 stars of the RR Lyrae type with periods between 0.3 and $I \cdot 2$ days. Sanford has observed several Cepheids and RV Tauri stars for velocity and spectral changes.

Io. Spectrophotometric studies are being carried on at a number of places, as well as studies in different wave-lengths by the use of selective filters or photocells. Thermoelectric radiometry, and photography in various colours, especially the infra-red, are being pushed.

At the Wilno Observatory the programme of obtaining accurate photographic and photovisual light curves of ten Cepheids from simultaneous exposures is nearing completion. A general survey of all Cepheids brighter than $\mathrm{II}^{\mathrm{m} \cdot 5}$ at minimum and north of declination $-15^{\circ}$ to determine the photographic and photovisual magnitudes at maximum and minimum has also been undertaken. This programme will include determination of the magnitudes of comparison stars.

II. Great numbers of variables are being discovered-by Luyten in his comparison of plates for proper motions; by Hoffmeister with his patrol plates; by astronomers in Leiden from plates taken in Johannesburg; by O'Leary and O'Connell in Sydney, Australia. At the Harvard Observatory a relentless search for variables is being made in the attempt to find the distances of star clouds. Their programme includes studies of variables in southern globular clusters, in fields of the Milky Way, in the anti-centre region especially where the presence of numerous external galaxies indicates small total absorption, and in a region close to the galactic centre; surveys through the galactic window in longitude $300^{\circ}$, and in fields on the borders of the Milky Way, galactic latitude $\pm 20^{\circ}$ to $\pm 30^{\circ}$, this to be combined with a survey of the same zones for external galaxies to indicate 
the distribution of absorbing matter; and surveys in large selected areas to provide adequate statistics on the frequencies of variables. At the Nantucket Observatory Miss Harwood seeks out variables in and about the Scutum Cloud on a similar programme.

I2. Several members deplore the lack of communication lines for the exchange of information about programmes. They suggest that individuals and institutions interested in the same type of variable or in closely related problems of variable star investigation may be encouraged to form groups within which exchange of information will be practicable. (Kukarkin) If, further, clearing houses for spectroscopic and photometric research were selected, then any point about light variation or spectrum peculiarities could be communicated to these headquarters, and plans for proper observation and study could be made; eventually, perhaps, all observations of a certain object to be sent to one place for discussion. VV Cephei and $\zeta$ Aurigae are mentioned as cases in point. (Gaposchkin)

In the meantime it seems safe to urge that present facilities be better utilized: the extension of personal correspondence, to the mutual improvement and completeness of individual programmes; the prompt publication of observations; and the following of the good example of many observatories in giving their programmes, both current and future, with sufficient detail in their annual reports. One member asks particularly for photographic programmes-the fields under observation, with magnitude limit, exposure time, and area of field. (Gaposchkin) As to the collection of the material on any one star, it is questionable whether anything more can be done than to follow the present practice of advertising in a journal one's willingness to act as a receiving agent.

In this connection it may be mentioned that Commission 30 has a sub-committee for co-operation in radial velocity observation and that its chairman, $\mathrm{Dr} \mathrm{J}$. H. Moore, invites variable star workers to suggest stars that may profitably be placed on the radial velocity programmes of the observatories which report to this subcommittee: Berlin-Babelsberg, David Dunlap, Lick, Michigan, Mount Wilson, Pulkova; Simeis, Victoria, and Yerkes.

13. There is doubtless general agreement in principle that writers should aim to make it easy for readers to identify stars for observation and to trace their history in the literature. A little thought will suggest what will be helpful to the readercharts, accurate positions, magnitude ranges, conventional time reckoning, designations as variable stars. While co-ordinates of the customary accuracy are of course essential, they do not always serve to distinguish the variable when it is one of a close group of stars. Furthermore, if the discoverer will prepare an inexpensive chart once for all, it will not be necessary for each observer to hunt out a Franklin-Adams chart and scale off the position. Writers are also urged to give the usual designation as a variable star. If the star has only a provisional designation, the name of the constellation and the position should be given. Quite a number of people might be saved quite a lot of time in tracing cross references to the (fictitious) "announcement of spectrographic observations of the eclipsing variable HD 216014". Since to every article on variable stars published by spectroscopists there are probably at least one hundred articles or notes published by observers of brightness, it is hardly fair to turn this round and expect every writer on variable stars to give $\mathrm{HD}$ or Boss numbers. Another case typical of those that may cause much woe to many people is a statement published in 1937 that 470.1934 has just been found to be of the $\beta$ Lyrae type-no co-ordinates, no references, and perhaps even no constellation given. 
I4. Another bewildering experience to the keeper of a special card catalogue is to find practically the same material published at intervals in several places, not, however, in reprint form, but sufficiently different in appearance to make it necessary to look each article through carefully lest something be missed.

I5. One member urges the lasting value of a publication containing elements and charts only and published at intervals greater than a year, as opposed to annual ephemerides.

I6. The need is pointed out by Jacchia for more charts and sequences for faint stars, especially of the $U$ Geminorum type, that could be reached by several amateurs who have access to fairly large telescopes. McLaughlin, on the other hand, deplores the necessity of using for some of the bright variables sequences determined under non-uniform conditions.

I7. Attention is again called, by McLaughlin, to the value of a survey with a fast spectrograph of the spectra of variable stars not included in the HD or its extensions. Joy writes on this same subject, pointing out that in the $K u E$ for I937 about 700 stars are listed as brighter than IIth magnitude and north of declination $-20^{\circ}$ which could be readily observed for spectral type with moderatesized instruments. He calls attention to the particular advantage of simultaneous spectrographic and photometric observations where there is irregularity in the period or shape of the light curve.

I8. Very little has been done in observing the eclipsing stars fainter than $9^{\mathrm{m} \cdot 5}$ at maximum for spectral type, and of course still less has been done for radial velocities.

I9. The position of the rapid irregular RR Tauri stars among the other variables is still rather puzzling, especially on account of the lack of spectroscopic data. As all of them (RR Tau, RW Aur, SY Cnc, UX Ori, RY Ori) reach at least the roth magnitude at maximum, it would not be very difficult to get detailed spectrograms of them. A more detailed description of the spectrum of V Sagittae (Ob) is highly desired. (Jacchia)

20. Shapley wishes to have the careful spectrophotometry of brighter variables of the Cepheid, irregular, and long-period classes emphasized.

2I. It seems very desirable to test by proper observation whether in the irregular variables of spectral classes $\mathrm{S}$ and $\mathrm{N}$ the small change in brightness is not mainly due to irregular variations in the band absorption. Attention is again directed to the importance of systematic photoelectric and spectrographic observation of the Be stars. (Potsdam, Berlin-Babelsberg)

22. The German group also refers again to the necessity of each observer determining his reaction to colour in a quantitative manner, suggesting that a list of circumpolar star pairs of different spectral type and of known intensity distribution or at least of known spectral type be selected for common observation.

23. More observations of eclipsing variables in red and infra-red are urged. Kopal points out that there is probably often a difference of form in a small, visually bright component, and a large, visually faint component. If the two are more nearly equalized in brightness by observation in longer wave-length, one may expect to get evidence of the tidal distortion of the redder component; to find the two minima more nearly equal in depth; and to standardize the darkening to a definite wave-length. He expects soon to announce results of such investigations.

24. From the same member comes the proposal that (I) a systematic photographic search be made for variables in the regions surrounding all globular clusters which are known to be rich in cluster variables; (2) that a similar search be 
made around clusters in which there are few or no variables; (3) that radial velocities and proper motions of as many galactic short-period Cepheids as possible be determined.

25. Joy points to the good use that could be made of an approximate classification by type and thinks that experienced discoverers should be encouraged to guess at the type from characteristics such as range, spectrum, and colour.

26. In order to make ready distinction between ordinary novae and supernovae it is suggested that the designation of the latter contain the number of the nebula or cluster in which they appear. (Helen Sawyer Hogg)

27. Closer cooperation is desirable between those who use observational material in memoirs, monographs, etc., and those who have made the observations. Authors should consult, when possible, with the observers concerning any difficulties which may occur in the interpretation of the observations. Values given with the observer's name should be those furnished by the observer himself or authorized by him. Authors should give full particulars of the material on which their studies are based including the name of each observer, place of observation, instruments used, total number of observations by each person and period covered. (Ryves)

28. The method of observing variable stars by visual estimates is capable of giving very good results when the field is good; but when the field is bad (e.g. when there are gaps in the sequence, bright stars in the field, or another star very near the variable) observation is difficult and results are of inferior quality. Since the number of long-period variables is very great, and it seems unnecessary to observe them all continuously, it is recommended that stars with inferior fields should not be regularly observed by this method. (Ryves)

It is proposed that a yearly subsidy be granted, as in former years, toward the publication of the Cracow Ephemerides of Eclipsing Binaries (SAC).

Raymond S. Dugan President of the Commission 